How to reference this article Kowalczyk, M.E. (2021). Le lettere di Tomasz Kajetan Węgierski scritte durante il viaggio del 1779 attraverso il Veneto. Italica Wratislaviensia, 12(2), 69-85.

DOI: http://dx.doi.org/10.15804/IW.2021.12.2.04

\author{
Małgorzata Ewa Kowalczyk \\ Uniwersytet Wrocławski, Polonia \\ malgorzata.kowalczyk@uwr.edu.pl \\ ORCID: 0000-0001-7623-1567
}

\title{
LE LETTERE DI \\ TOMASZ KAJETAN WEGGIERSKI \\ SCRITTE DURANTE IL VIAGGIO DEL 1779 \\ ATTRAVERSO IL VENETO
}

\author{
TOMASZ KAJETAN WEGGIERSKI'S LETTERS \\ FROM HIS TRAVELS \\ AROUND VENETO IN 1779
}

\begin{abstract}
In the rich prose of Tomasz Kajetan Węgierski (1755-1787), a talented poet and satirist, a special place is occupied by a French-language diary. This diary was in the form of letters written during a journey around Italy in 1779 . His recipient was a certain Julia, a friend and lover of the poet living in Paris. His letters survived in the Manuscript Section of the Jagiellonian Library in Krakow, though, unfortunately, as an incomplete copy only: just seven letters, written in November and December from Verona, Vicenza, Padua, and Venice, have been preserved entirely. We also have the beginning of an eighth letter, written from Venice. Furthermore, we have the poet's comments on several missing letters, four of which refer to the city on the lagoon and one to Ferrara. There is also a fragment of a letter from Rome devoted to the catacombs. From its content, we learn that the poet intended to return to the city of St. Mark to see the wedding ceremony of the Venetian Republic with the sea. The aim of this article is to present the motivations that prompted the poet to leave the PolishLithuanian Commonwealth, as well as to analyse his impressions from his trip around Veneto. It is worth noting that this region was only a stage, though a very important one, on the poet's route leading to the capital of the Papal States. This Italian region fascinated, surprised, and shocked the Pole, and in his notes, which are full of anecdotes and jokes, we find extensive descriptions of the history and culture of Verona, Vicenza, Padua, and Venice. Also included are more-detailed characteristics of the inhabitants and their customs, as well as all kinds of information about everyday life on the road. Weggierski's narrative, though different in many respects from the travel descriptions of his predecessors, is quite typical of the century in which he lived.
\end{abstract}

Keywords: Tomasz Kajetan Węgierski, Veneto, travels, letters, daily life 
Tomasz Kajetan Węgierski (1755-1787) è una delle figure più inte1 ressanti e al contempo controverse dell'Illuminismo polacco. Questo poeta di talento, satirico e scrittore prematuramente scomparso, non cessa di suscitare interesse fra gli studiosi, come dimostrano le ristampe delle sue poesie (Węgierski, 2002, 2007), alcune monografie concernenti la sua opera (Bobrik, 1981; Kaczyński, 2001; Stasiewicz, 2012), nonché numerosi articoli (Czyż, 1992, 2004; Kaczyński, 2003, 2011; Ordzowiały-Grzegorczyk, 2011; Ziontek, 2016; Konończuk, 2017).

Nella ricca produzione in prosa di Tomasz Kajetan Węgierski uno posto di rilievo è occupato dai diari di viaggio in francese, redatti sotto forma di epistole, scritte durante i suoi viaggi in Italia, nei Caraibi e nell'America del Nord negli anni 1779-1783. La sua destinataria era una certa Julia, amica parigina e amante del poeta. Le lettere di Węgierski, scritte nel corso dei suoi viaggi per il mondo, si sono conservate nelle collezioni della Sezione Manoscritti della Biblioteca Jagellonica di Cracovia, purtoppo sotto forma di una copia incompleta (BJ, cc. 1r-165v). Un'altra descrizione delle sue lettere si trovava nella biblioteca dei Chreptowicz a Szczorse ancora agli inizi del XX secolo. Attualmente, purtoppo, tale copia si considera andata perduta (Kaczyński, 2001, pp. 10-11). Di entrambe le trascrizioni si avvalse Stanisław Kossowski, che nel 1908 pubblicò sul Przewodnik Naukowy $i$ Literacki (Guida Scientifica e Letteraria), le lettere dei viaggi extraeuropei di Węgierski in traduzione polacca (Kossowski, 1908). Per motivi ignoti egli rinviò l'edizione delle note di viaggio nella Penisola Appenninica e non vi mise più mano. Non sappiamo, pertanto, in che misura il manoscritto della biblioteca di Szczorse colmasse le lacune di quello jagellonico per quanto riguarda l'Italia.

Ci sono pervenute sette lettere complete, scritte nel novembre e nel dicembre del 1779 da Verona, Vicenza, Padova e Venezia, nonché l'inizio di un'ottava, anch'essa datata da Venezia (BJ, cc. 2r-32v). Inoltre, disponiamo dei commenti di Węgierski ad alcune lettere mancanti, di cui quattro riguardano la città della laguna e uno Ferrara (BJ, cc. 41r44r). Si è conservata anche una parte di una lettera scritta da Roma, dedicata alle catacombe (BJ, cc. 46r-49r). Da essa veniamo a sapere che il poeta intendeva tornare nella città di San Marco per assistere alla 
cerimonia dello Sposalizio della Repubblica di Venezia con il Mare, che cadeva in concomitanza con la festa dell'Ascensione.

Il diario epistolare italiano di Tomasz Kajetan Węgierski non è ancora stato oggetto di indagini scientifiche. Le lettere incomplete hanno suscitato e suscitano scarso interesse da parte degli studiosi. Lucjan Siemieński se ne servì per scrivere la biografia del poeta (Siemieński, 1850), per Mieczysław Brahmer costituirono la base per ricostruire la vita quotidiana dei viaggiatori polacchi a Venezia (Brahmer, 1936; 1980, pp. 215-218), mentre Alojzy Sajkowski vi attinse la lista dei libri che Węgierski studiava durante il suo viaggio nel Veneto (Sajkowski, 1973, pp. 202-203). Le lettere sono state trattate più ampliamente da Paweł Kaczyński nella monografia dedicata alla prosa di Węgierski, nonché nell'articolo sulle città tedesche ed italiane viste con gli occhi del poeta (Kaczyński, 2001, pp. 105-130; 1993). Attualmente i diari di Węgierski sono tradotti dal francese al polacco e predisposti per un'edizione scientifica da Małgorzata Ewa Kowalczyk e Anna Pikor-Półtorak.

Lo scopo del presente articolo è quello di presentare le ragioni che indussero il poeta nel 1779 a lasciare la Confederazione polacco-lituana per l'Italia, nonché di analizzare il contenuto delle sue lettere, scritte durante il soggiorno in Veneto. Occorre sottolineare come tale regione costituisse solo una tappa, per quanto importante, sulla via che conduceva Wegierski nella capitale dello Stato Pontificio. La Venezia Euganea affascinava il polacco, lo sorprendeva e scioccava. Nelle sue annotazioni, piene di aneddoti e di facezie, troviamo ampie descrizioni della storia e della cultura di Verona, Vicenza, Padova e Venezia, estesi profili degli abitanti e delle loro usanze, ed anche le più svariate informazioni sulla vita di tutti i giorni, raccolte lungo la via. La narrazione di Węgierski, pur discostandosi per diversi aspetti dalle descrizioni di viaggio dei suoi predecessori, è tipica dell'epoca in cui visse. Teresa Kostkiewiczowa definì il suo diario epistolare di viaggio "vicinissimo alla maniera tradizionale di annotare le impressioni di viaggio" (Kostkiewiczowa, 1975, p. 362). Un tentativo di ricostruire il modo di vedere il Veneto da parte del poeta illuminista polacco contribuirà certamente ad una migliore conoscenza della storia di questa regione. 
Tomasz Kajetan Węgierski nacque nel 1755 nel villaggio di Grabowiec, situato nei pressi di Bielsk Podlaski. Era figlio di Aniela Paprocka (1733-1819) e Tomasz Węgierski (1722-1788), starosta di Korytnica. Il poeta e viaggiatore venne alla luce in una famiglia benestante e socialmente ben relazionata. I suoi genitori ingrandivano di continuo la loro proprietà, acquistando sempre nuove terre nel Podlasie. Inoltre, essi erano buoni amici del comes castellanus di Cracovia, Jan Klemens Branicki (1689-1771) e di sua moglie Izabella Poniatowska (1730-1808), sorella del re Stanislao Augusto. Alla corte dei Branicki di Białystok, detta "la Versailles polacca", il giovane Węgierski soggiornava regolarmente. Proprio là, nel 1772, all'età di diciassette anni, fu ritratto da Augustyn Mirys (1700-1790), pittore di corte dei Branicki (Konończuk, 2017, pp. 237-241).

Tomasz Kajetan fu educato attentamente in casa e ricevette una solida educazione scolastica. I genitori fecero in modo che fin dai primi anni studiasse il francese, assumendo probabilmente nella loro tenuta di Grabowiec un precettore madrelingua. Nel 1764, a nove anni, fu mandato a studiare al Collegium Nobilium di Varsavia, scuola d'élite dei gesuiti, dove rimase fino al 1771. Il padre superiore dell'istituto era Karol Wyrwicz, eminente pedagogo e geografo, mentre uno dei docenti era Adam Naruszewicz, eccellente poeta, storico e traduttore. Nel 1769 Węgierski si esibì in un saggio sulla Turchia, e poco prima di concludere il ciclo scolastico debuttò su importanti giornali polacchi come autore del discorso di benvenuto rivolto al re Stanislao Augusto in visita al collegio, nonché in qualità di traduttore dal francese (Puchowski, 2007, pp. 60, 439-440). Negli anni successivi intorno alla produzione letteraria di Węgierski si creò un'atmosfera di scandalo e scalpore. Il giovane scrittore attraverso una pubblicistica sia in prosa che in versi, piena di malignità e allusioni, nota in tutta la Polonia, criticava la degenerazione della società e della politica, mettendo spietatamente a nudo l'apparente moralità di personaggi famosi (Kaczyński, 2001, pp. 19-51). Il ridicolo gettato sui magnati, le loro famiglie e gli amici fece sì che Węgierski fu escluso dai circoli mondani di Varsavia. Nel 1777 perse anche l'incarico 
di cancelliere presso il Dipartimento di Giustizia del Consiglio Permanente, ottenuto nel 1775. La sua penna pungente ed i giudizi intransigenti gli procurarono molti nemici accaniti. Di conseguenza, quando nel 1778 cercò di candidarsi a deputato del Sejm della Confederazione polacco-lituana, gli fu difficile ottenere l'appoggio del re e di altre persone altolocate (Konończuk, 2017, pp. 243-244). Le speranze deluse e gli insuccessi personali ebbero un peso determinante nella decisione di Węgierski di lasciare la Polonia. Come ha osservato Paweł Kaczyński, "forse ritenne che all'estero avrebbe trovato il modo di distinguersi, o semplicemente aspettava che passasse lo sfavore del re e di alcuni signori (e delle loro mogli e amanti)" (Kaczyński, 2001, p. 92). Nel 1779 Tomasz Kajetan Węgierski lasciò la patria per non farvi mai più ritorno. Il poeta morì nel 1787 a Marsiglia, all'età di soli trentun anni.

La partenza di Węgierski dalla Polonia aveva il carattere di emigrazione temporanea. Sappiamo che progettava di rientrare in $\mathrm{Pa}-$ tria e di candidarsi nuovamente al Sejm (Konończuk, 2017, p. 244; Kaczyński, 2011). Aggiungiamo che la decisione di abbandonare la Patria era dovuta non solo alle delusioni e alle difficoltà della vita, bensì anche al desiderio di visitare diversi paesi e a motivi mondani. Il poeta intraprese il viaggio all'estero in compagnia di Kajetan Potocki (ca.1749-1802), starosta di Urzędów, diretto a Spa, celebre località d'incontro dell'elegante società europea. Il pretesto per il soggiorno a Spa erano le virtù curative delle locali acque minerali, ma in realtà l'attrattiva della stazione termale era innanzitutto di natura mondana (Wdowik, 2014, pp. 217-228). "Lasciai Spa, versando lacrime sulla compagnia là rimasta, sul denaro speso ed il tempo sprecato. Mentre le prime due cose si possono recuperare, la terza, purtoppo, è svanita per sempre" - scriveva Węgierski in una lettera all'amica francese (BJ, c. 2r).

Dalla stazione termale alla moda proseguì egli verso il Veneto, scegliendo l'itinerario che toccava Aquisgrana, Colonia, Magonza, Francoforte, Augusta, Innsbruck, Trento e Rovereto. La traversata delle Alpi nei pressi del Brennero dove passava una delle vie di comunicazione europee più importanti, ma anche più difficili, suscitò nel poeta emozioni negative. Esperto nel peregrinare per i terreni pianeggianti e collinari della Confederazione polacco-lituana, egli si sentiva a disagio 
in montagna. Niente di strano: si trattava allora di regioni ancora poco conosciute e scarsamente abitate dall'uomo. La loro topografia era fonte di svariati pericoli per i viaggiatori (Woźniakowski, 1995; Kowalczyk, 2004). Węgierski descrisse così all'amica le impressioni del suo arrivo in Veneto: "Ero tediato dalle montagne, perciò gioivo di essermi ritrovato in una ridente pianura" (BJ, c. 7r).

Nel XVIII secolo arrivare dalla Confederazione polacco-lituana nella Penisola Appenninica richiedeva un grosso sforzo. Malgrado ciò l'Italia era un punto fermo ed estremamente importante sulla mappa dei viaggi dei polacchi d'altri tempi (Barycz, 1965; Sajkowski, 1973; Chachaj, 1998, pp. 19-131; Kowalczyk, 2005, pp. 55-90; Wrześniak, 2010, 2013; Markiewicz, 2011, pp. 28-31). La si visitava per allargare i propri orizzonti, sia estetici che filosofici, soddisfare esigenze spirituali e aspirazioni intellettuali, nonché per il piacere stesso di visitare. L'Italia costituiva indiscutibilmente il centro dell'arte, della cultura e della scienza del mondo moderno. Chiunque volesse attingere alle fonti della civiltà europea vi si recava. Per i giovani aristocratici di tutta l'Europa l'Italia era una tappa obbligatoria del viaggio d'istruzione del tipo grand tour. Il suo scopo era affinare le buone maniere, completare la formazione nelle sue università, e anche allacciare contatti fruttuosi con l'aristocrazia internazionale. Nella seconda metà del XVIII secolo il grand tour della nobiltà perse progressivamente il suo carattere conoscitivo ed educativo a vantaggio di quello rappresentativo e dilettevole (Bratuń, 2003, pp. 72-74). Iniziò a farsi largo la convinzione che valeva la pena di andare a Venezia, Bologna, Firenze, Roma o Napoli per il solo fatto che vi erano gli altri. Nell'epoca dell'Illuminismo era di moda la corsa alla realizzazione dei sogni turistici. Era essa ad imporre il viaggio in Italia a chiunque pretendesse al titolo di persona civile (Kowalczyk, 2019, pp. 50-74). In tale novero non poteva mancare Tomasz Kajetan Węgierski. Del resto egli era perfettamente preparato a visitare la Penisola Appenninica. Nelle sue lettere spesso faceva riferimento a viaggiatori precisi, di cui teneva nei bagagli le relazioni di viaggio stampate, note in tutta Europa. Vi erano fra l'altro le opere di François Maximilien Misson (Voyage d'Italie, Amsterdam 1743), Joseph Jérôme Lefrançois de Lalande (Voyage d'un français en 
Italie, fait dans les années 1765 et 1766, Parigi 1769) o Pierre-Jean Grosley (Observations sur l'Italie et sur les Italiens..., Londra 1770). Occorre aggiungere che Węgierski conosceva abbastanza bene l'italiano, poiché si interessava vivamente dei suoi dialetti (Sajkowski, 1973, p. 203).

Il poeta polacco giunse nel Veneto nella prima metà di novembre del 1779. Portava con sé alcune lettere credenziali che dovevano introdurlo nella società mondana di Verona, Vicenza, Padova e Venezia. In epoca moderna, quando un cognome di per sé non diceva abbastanza, occorreva avere lettere di raccomandazione e affidabili conoscenze sul posto che permettessero di entrare nei saloni eleganti (Mączak, 2001, p. 160). Węgierski così scriveva il 13 novembre 1779 da Verona all'amica parigina: "Una [delle lettere credenziali] era destinata stando alle parole di chi me l'aveva consegnata - alla dama più bella, intelligente ed altamente illuminata di questo paese" (BJ, c. 7r). Non rimase deluso da questa conoscenza. Dopo aver fatto visita alla dama, affermò: "A quella cui sono stato raccomandato non manca né intelligenza né fascino. Declama splendidamente e perfino compone versi ella stessa" (BJ, c. 12r). Tomasz Kajetan trascorse a Verona una quindicina di giorni. In altre due lettere a Julia del 21 e 23 novembre 1779 egli avanzò svariate ipotesi sull'origine della città, elencò i personaggi celebri in essa nati e morti, descrisse la situazione politica ed economica del momento, la vita teatrale ed indicò i luoghi degni di essere visitati: il giardino Giusti, l'anfiteatro, i "gabinetti di storia naturale e antica", le chiese di Giorgio in Braida e San Tommaso Becket, fornite di "qualche bel quadro" di artisti rinascimentali (BJ, c. 10v). Tipico di Węgierski era il fatto di visitare le opere d'arte di cui in precedenza non sapeva dire niente di interessante a parte il nome dell'autore. D'altra parte egli era un attento osservatore della vita quotidiana. E così risulta che di Verona e dei suoi abitanti non aveva una buona opinione. A dire il vero era affascinato dalla bella posizione della città sul fiume Adige, dalla sua grandezza e densità di popolazione, ma la riteneva molto provinciale. Dei veronesi scriveva senza mezzi termini: "I loro abiti sono sporchi e vecchi, la servitù mal vestita, i mobili rovinati e da tempo fuori moda. La gioventù non è educata, ad eccezione di alcuni 
giovani che i viaggi hanno reso più garbati. Nel complesso tutto ciò non fa buona impressione e sa di provincia" (BJ, c. 11v).

Da Verona Węgierski si diresse a Vicenza. Al profilo della città e dei suoi abitanti dedicò una lettera, scritta il 25 novembre 1779, in cui sottolineò che Vicenza deve la sua fama architettonica ai progetti di Andrea Palladio, maestro del Rinascimento, che costruì la maggiore parte delle residenze della nobiltà locale. Grazie alle lettere credenziali il poeta polacco conobbe un certo nobiluomo che gli mostrò in città tutto ciò che era degno di esser visto. "Senza fretta vedemmo palazzi, giardini, chiese, piazze" - scrisse in una lettera all'amica parigina (BJ, c. 13r). Con stupore scoprì che la maggioranza delle case progettate dall'eminente architetto rinascimentale nonché teorico dell'architettura aveva magnifiche facciate ed ingressi maestosi che conducevano ad ambienti sporchi, tanto da sembrare "soffitte", dove i divani erano tutti rappezzati o a brandelli. Notò l'indigenza della nobiltà locale anche durante il noto svago serale che consisteva nel girare in carrozza sulla spianata detta Campo Marzio. "Mi fa un gran pena il manto erboso strapazzato da sette o otto vecchi macinini, indebitamente chiamati calessi. (...) Proprio qui gli abitanti di Vicenza ogni giorno danno mostra reciproca della loro povertà" - concluse Węgierski (BJ, cc. 14v-15r). Il resto del soggiorno fu dedicato dal poeta alla visita del Teatro Olimpico e della celebre chiesa mariana detta Madonna del Monte, situata alla periferia della città, visitata non per motivi religiosi, ma per il piacere di vedere un quadro di Paolo Veronese, collocato nel refettorio del convento, nonché per godere dello splendido panorama che si dischiude dalla collina. Si recò anche alla famosa Villa Almerico-Capra, detta La Rotonda, eretta nei pressi della città su progetto del Palladio. Il padrone della residenza accompagnò con piacere il viaggiatore polacco negli interni dell'edificio. "Salimmo fin sul tetto ed ebbi il diletto di guardare da lassù i dintorni di Vicenza, pieni di case stupende e disseminati di gelso bianco. Quell'ampia veduta era chiusa da ridenti colline, disposte ad anfiteatro" - scriveva il poeta (BJ, c. 14r). Osservò al contempo che non si sarebbe espresso sulla situazione politica e commerciale di Vicenza, "poiché entrambe le cose non lo meritano. Il governo è identico a quello di qualsiasi altra città sottoposta al potere veneziano" (BJ, c. 15r). 
Padova, dove trascorse qualche giorno e scrisse all'amica due lettere, datate 29 novembre e 2 dicembre 1779, fece a Węgierski un'impressione deprimente. Per il poeta polacco la città - un tempo celebre per l'università che attirava frotte di studenti da diversi paesi europei - era triste, sporca, fangosa, scarsamente popolata. Osservazioni simili furono riportate qualche anno dopo, nel 1786, da August Fryderyk Moszyński (1731-1786), architetto-dilettante, esperto di scienza e di arte, collezionista e conoscitore del teatro (Łukaszewicz, 2021, pp. 127-131). Scriveva Tomasz Kajetan Węgierski nella lettera del 29 novembre all'amica parigina: "A Padova si vedono ad ogni angolo le tracce dell'antico splendore. L'enorme sala del municipio, la chiesa di Sant'Antonio, quella di Santa Giustina o l'edificio dell'università testimoniano di come fosse Padova un tempo" (BJ, c. 19v). L'epistole si apre con una lunga digressione sulla vita e l'opera di Francesco Petrarca, ispirata dall'aver visto un suo ritratto nella sagrestia del duomo di Padova, nonché dalla visita al cimitero di Arquà, dove fu sepolto l'autore dei sonetti a Laura. Secondo Węgierski le opere di Petrarca sono splendide, ma contengono troppi pensieri contorti ed astrusi. Riflettendo sul genere di sentimenti che la "bella" Laura nutriva, il quale "non era una bellezza", giunse alla conclusione che fosse più una civetta che non una tenera amante (BJ, c. 16v; Sajkowski, 1973, pp. 202-203).

Descrivendo Padova Węgierski rievocò la storia della sua fondazione da parte di Antenore, basandosi sull'autorevolezza di Virgilio e di Livio. Andò perfino a vedere la Tomba che i padovani consideravano il luogo in cui riposava il valoroso troiano. Węgierski era del tutto cosciente del fatto che gli studiosi da tempo avevano fatto ricredere gli abitanti su "quell'allettante errore" (BJ, c. 19r). Soggiornando in città per alcuni giorni visitò i monumenti, $\mathrm{i}$ gabinetti delle meraviglie, le piazze, frequentò le caffetterie, ma soprattutto osservò la gente e le sue usanze. Il fatto di possedere lettere credenziali gli assicurò "il piacere di partecipare" alle fastose nozze della figlia di Giovanni Alvise Mocenigo, patrizio veneziano e mecenate d'arte. Il viaggiatore polacco notò che $\mathrm{i}$ veneziani visitavano in massa Padova e - così come i suoi abitanti - trovavano gusto a girare in carrozza per il Prato della Valle. "È un'enorme piazza circondata da statue di tutti coloro che intendono 
pagarsele" - scrisse con sarcasmo, aggiungendo: "Se solo mi ritenessi degno di trovarmici, potrei regalarmi una statua così" (BJ, cc. 24v-25r).

A parere di Węgierski la chiesa di Santa Giustina era uno dei monumenti sacri più belli di tutta l'Italia. Minore impressione fece su di lui la Basilica di Sant'Antonio. Egli si espresse con scetticismo sui numerosi e ricchi ex-voto della cappella contenente le reliquie del Santo. Egli si faceva anche beffe in modo esplicito del valore delle spoglie mortali dei santi che - come cercavano di fargli credere - emanavano un olezzo dolce e piacevole, una sorta di "santo profumo". Węgierski scrisse all'amica: "Mi sono recato due volte nella cappella, ma era sempre chiusa. Comunque non avrei sentito niente perché avevo il raffreddore" (BJ, c. 20v). Il poeta era invece affascinato dagli affreschi che abbellivano il santuario, sebbene non sapesse scrivere di essi niente di particolare. Notò tuttavia che nella Basilica di Sant'Antonio "si trova una gran quantità di tombe e di epitaffi, e fra essi molti dedicati ai polacchi" (BJ, c. 20v).

Nelle sue annotazioni molto spazio era dedicato all'Università di Padova. In una lettera a Julia scriveva: "Come potrei parlarti di Padova senza menzionare la sua università?” (BJ, c. 22v). Non era però entusiasta della sua passata fama, che attirava in città frotte di giovani d'oltralpe. Valutava l'eredità dell'ateneo patavino dal punto di vista dello stato della scienza a lui coeva. Riteneva che un tempo l'università, in cui dominava la teologia morale in senso tomistico e la filosofia di Aristotele, era famosa perché la gente era ignorante e bastava poco per soddisfare le sue aspettative. La maggioranza degli ex-professori era da lui definita tout court "degenerati, che erano ammirati senza sapere che cosa volessero" (BJ, c. 23r). Solo la riforma dell'ateneo, ai suoi tempi in atto, era oggetto di stima. "Attualmente l'università non è più tanto grande né famosa, benché persone molto intelligenti vi insegnino molte cose utili" - scriveva il 29 novembre 1779 (BJ, c. 23r).

Il coronamento del viaggio di Węgierski attraverso la regione Veneto fu il soggiorno nel suo capoluogo. Nell'epoca dell'Illuminismo Venezia si piazzava alla testa dell'elenco delle città italiane frequentate più di buon grado (Biliński, 1968; Tenenti, 1985; Kowalczyk, 2014). Frotte di stranieri giungevano a Venezia per ammirarne l'eccezionale posizione, 
i numerosi monumenti dell'arte e dell'architettura, infine per deliziarsi dell'irripetibile atmosfera della città. Malgrado si siano conservate solo due lettere (la seconda solo in parte) scritte da Węgierski a Venezia, vi rinveniamo una marea di osservazioni. La lettera del 4 dicembre 1779 inizia con questa dichiarazione del poeta: "Volevo guardare Venezia come se non ne avessi mai sentito parlare. Le descrizioni che avevo letto, ciò che si dice ai viaggiatori, me ne avevano dato un'immagine falsa. Bisogna che - usando cautela e opponendomi a me stesso - non esprima opinioni che possano essere il frutto di stereotipi radicati” (BJ, c. 27r). Węgierski riteneva, come del resto molti illuministi, che solo la conoscenza diretta del paese e della gente che lo popolava avesse valore (Kowalczyk, 2005, pp. 60-61). Era un sostenitore dichiarato dell'empirismo.

A Venezia gli piacque letteralmente tutto, a partire dalla mentalità dei suoi abitanti, per finire con il dialetto che considerava l'idioma più bello d'Europa. La gente era ciò che gli interessava sempre enormemente. Nella lettera a Julia scriveva: "Sebbene Venezia - per la sua posizione atipica - sia uno dei luoghi più sorprendenti al mondo, mi colpisce molto di più il morale di questa grande città, il suo governo, l'ingegno dei suoi abitanti, le loro usanze, le abitudini, il modo di vestire" (BJ, c. 27r). Fin dal primo giorno della sua permanenza a Venezia egli rimase affascinato dalla comunità dei gondolieri. Come scriveva scherzosamente, questi guidano "le carrozze veneziane" - barche strette, lunghe e nere. Un giovane gondoliere, tale Gandino, che servì il poeta durante il suo soggiorno in città, divenne il modello del ritratto collettivo di questo caratteristico ambiente. A parere di Węgierski, i gondolieri, che in città erano circa 25 000, erano persone molto affezionate al loro lavoro, sempre allegre e perfettamente al corrente di tutti gli scandali. I continui capricci delle signore e dei signori da loro serviti facevano sì che eseguissero qualsiasi compito senza la minima sorpresa. Era loro interesse essere discreti. Se uno di loro veniva cacciato di casa per pettegolezzi, non trovava lavoro altrove.

Il viaggiatore polacco rimase molto affascinato dalle veneziane. Le considerava fra le donne più belle d'Europa. Nelle sue lettere dedicò molto spazio alla descrizione del loro aspetto fisico, ai tratti del 
carattere, al modo di abbigliarsi, alla loro vita quotidiana, nonché alle loro usanze e costumi. La corsa al piacere, sosteneva Węgierski, cominciava per loro nella prima gioventù e durava fino alla morte. A suo avviso soprattutto le donne non si immaginavano che nel tempo libero ci si potesse occupare di qualcosa d'altro che non fosse fare all'amore. Le attività manuali quali il ricamo, l'uncinetto o la tessitura erano da loro considerate pietose. "Di tutte le fanciulle che conosco, queste sono le uniche spinte a concedersi dal temperamento o [dal desiderio] del piacere. Per sventare il tradimento dell'amante occorre essere sempre vigili e all'erta. L'unico modo è spiare, ragion per cui qui ciò è molto di moda. Le donne spiano i loro amanti, i mariti le mogli, gli amanti le loro amanti. In giro non si vedono che spie andare avanti e indietro" - scriveva Węgierski, aggiungendo: "in fin dei conti tali ricerche svelano sempre che il marito viene tradito, l'amante abbandonata e l'amante maschio smascherato. Dopo un attimo di disperazione, tutti si consolano creando nuove relazioni, che si infrangono tanto facilmente quanto le precedenti. Ecco in che maniera si svolge qui la vita amorosa" (BJ, cc. 29r-29v). I depositari dei segreti connessi a tali intrighi erano di solito i gondolieri perché il teatro della maggior parte degli appuntamenti d'amore segreti erano le gondole.

Parte integrante della vita a Venezia era il portare le maschere e lunghi mantelli. Questo stile dominava nelle calli della città specie durante il carnevale. Ciò portava con sé una grande libertà e svariate comodità. Mentre infatti nelle altre città d'Europa già il semplice abito tradiva lo status sociale di un individuo, a Venezia ciò era efficacemente impedito da maschere e mantelli. Un tale costume toglieva anche la preoccupazione di non essere vestiti adeguatamente o alla moda. Così ricchi e poveri si concedevano insieme i piaceri del carnevale. Si passeggiava per vicoli e piazze, si frequentavano le caffetterie e la sera si correva alle feste da ballo o ci si recava alle rappresentazioni teatrali. "Appena le porte dei teatri si dischiudono, la gente inizia a mettersi in maschera. Gli abitanti di Venezia possono mostrarsi a teatro solo in quel modo o con la toga. La mattina, a proprio piacimento, passeggiano con mantelli scarlatti o con le maschere. È l'unico abbigliamento consono" - scriveva Węgierski nella lettera dell'8 dicembre 1779 (BJ, c. 31v). A Venezia 
il carnevale pareva durare tutto l'anno, o almeno questa è l'impressione riportata dal poeta polacco (Brahmer, 1936).

Tomasz Kajetan Węgierski era un esperto viaggiatore ed un impeccabile osservatore, come dimostrano le note di viaggio attraverso il Veneto da lui lasciateci. Una delle caratteristiche peculiari dell'epoca illuministica era da un lato "la febbre di viaggiare", dall'altro "la furia di scrivere", come dicevano i contemporanei. Essi hanno lasciato dietro di sé una marea di diari, memorie e volumi di corrispondenza. Węgierski annotava le impressioni di viaggio nella forma, al tempo spesso usata, della lettera. La convenzione epistolare nobilitava la relazione di viaggio ed era pertanto utilizzata di buon grado. Questo derivava dalla convinzione secondo cui a certi generi letterari era assegnata la forma stampata, ad altri quella del manoscritto. La descrizione di viaggio sotto forma di lettere "meritava" la stampa (Dziechcińska, 1990). Si direbbe che Węgierski avesse in progetto di pubblicare le sue note di viaggio italiane, basate sulle osservazioni e sulle conclusioni tratte da ciò che aveva visto con i propri occhi e vissuto di persona. Egli confrontava le proprie esperienze con le opinioni degli autori delle guide turistiche moderne più in voga, accusandoli non di rado di cedere agli stereotipi e ai pregiudizi. E così, secondo il polacco, il celebre viaggiatore francese Lalande "consiglia ciò che non merita di essere visto e tralascia cose molto più interessanti" (BJ, c. 6r). Tuttavia, non vi è dubbio che Węgierski abbia attinto a piene mani a molte descrizioni fatte da altri viaggiatori, trascrivendo ad esempio la "fattografia" (Kaczyński, 2001, pp. 118-119).

Il poeta polacco era figlio dell'epoca dell'Illuminismo, che credeva nella forza della ragione umana ed era convinta delle illimitate possibilià dell'uomo colto. Di qui la presenza nella sua narrazione di una marea di osservazioni critiche sulla Chiesa cattolica, sui religiosi, e soprattutto sul valore delle reliquie. Węgierski era spiritualmente disposto in primo luogo a conoscere la Venezia Euganea dal punto di vista scientifico. Era particolarmente interessato alla storia, alla cultura e ai rapporti sociali, cui dedicò lo spazio maggiore. Era persuaso del valore didattico della storia e la teneva in considerazione nel tracciare l'immagine futura delle città venete. Nelle sue lettere non ci sono aride descrizioni di opere d'arte e di architettura, elenchi di iscrizioni, tombe o esemplari raccolti 
nei gabinetti di storia naturale. Vi si possono invece trovare, non di rado sotto forma di aneddoti, descrizioni alquanto interessanti delle usanze e della mentalità degli abitanti di Verona, Vicenza, Padova e Venezia, del loro aspetto, dei tratti del carattere, degli svaghi, delle abitazioni e anche della cucina. Il viaggio di Tomasz Kajetan Węgierski per il Veneto è un esempio della nuova concezione del visitare, propria dell'epoca moderna, che occorre definire "illuministica". La sua peculiarità consiste nel suo carattere elitario, e non si tratta qui affatto di elitarismo sociale, bensì intellettuale e di obiettivi ambiziosi. Questo tipo di viaggi contribuiva a dare forma ad una nuova visione del mondo, e perfino a cambiarlo.

\section{BIBLIOGRAFIA}

\section{Fonti manoscritte}

BJ [Biblioteca Jagellonica di Cracovia], ms. 5634, Lettres du feu comte Thomas Cajetan Wegierski Polonois, écrites à differentes personnes, pendant son séjour en Italie et son voyage en Amérique fait l'année 1783 au quel on a joint le journal, écrit par l'Auteur, sur la fin de ses jours...

\section{Letteratura in materia}

Barycz, H. (1965). Spojrzenia w przeszłość polsko-włoską. Wrocław: Zakład Narodowy im. Ossolińskich.

Biliński, B. (1968). Viaggiatori polacchi a Venezia nei secoli XVII-XIX. In L. Cini (Ed.), Venezia e la Polonia nei secoli dal XVII al XIX (pp. 341-417). Venezia-Roma: Istituto della Collaborazione Culturale.

Bobrik, N.P (1981). Polskij poet proswietitel - Tomasz Kajetan Wiengierskij. 1755-1787. Moskwa.

Brahmer, M.(1936). Węgierski w Wenecji. InF. Bielak(Ed.), Prace historycznoliterackie. Księga zbiorowa ku czci I. Chrzanowskiego (pp. 271-280). Kraków-Warszawa: Skład główny w Kasie im. Mianowskiego.

Brahmer, M. (1980). Powinowactwa polsko-włoskie. Z dziejów wzajemnych stosunków kulturalnych. Warszawa: PWN.

Bratuń, M. (2003). Ars apodemica. Narodziny - rozwój - zmierzch. In P. Kowalski (Ed.), Wędrować, pielgrzymować, być turystą. Podróż w dyskursach kultury (pp. 67-76). Opole: Wydawnictwo Uniwersytetu Opolskiego. 
Chachaj, M. (1998). Związki kulturalne Sieny i Polski do końca XVIII wieku. Staropolscy studenci $i$ podróżnicy $w$ Sienie. Sieneńczycy $i$ ich dzieła $w$ Polsce. Lublin: Wydawnictwo Uniwersytetu Marii Curie-Skłodowskiej.

Czyż,A.(1992). Ten, który płynie „nałasce fal”'(Droga duchowa Węgierskiego), Ogród, 3/4, 212-235.

Czyż, A. (2004). Podlaska ojczyzna Tomasza Kajetana Węgierskiego. In I. Kadulska, \& R. Grześkowiak (Eds.). Od liryki do retoryki. W kregu słowa, literatury $i$ kultury. Prace ofiarowane profesorom Jadwidze $i$ Edmundowi Kotarskim. Gdańsk: Wydawnictwo Uniwersytetu Gdańskiego.

Dziechcińska, H. (1990). „Podróż” w druku i rękopisie. In H. Dziechcińska (Ed.), Staropolska kultura rękopisu (pp. 113-122). Warszawa: IBL PAN. Kaczyński, P. (1993). Niemieckie i włoskie miasta w oczach oświeconego Polaka (Listy T.K. Węgierskiego z podróży po Niemczech i Włoszech). Prace Historycznoliterackie, 16, 87-103.

Kaczyński, P. (2001). Niedokończona podróż. Proza Tomasza Kajetana Wegierskiego. Studia i przekroje. Wrocław: Wydawnictwo Uniwersytetu Wrocławskiego.

Kaczyński, P. (2011). Kłopoty z komunikacją, albo jak nie mówić do władców. Niepublikowane listy Tomasza Kajetana Węgierskiego do Stanisława Augusta. Pamiętnik Literacki, 4, 221-228.

Konończuk, W. (2017). Pożyteczny obywatel z Grabowa, czyli nieznane karty biografii Tomasza Kajetana Węgierskiego. Pamiętnik Literacki, 3, 235-250.

Kossowski, S. (1908). Z życia K. Węgierskiego. Pamiętniki i listy. Przewodnik Naukowy i Literacki, 36 (1-12).

Kostkiewiczowa, T. (1975). Klasycyzm, sentymentalizm, rokoko. Warszawa: Państwowy Instytut Wydawniczy.

Kowalczyk, M.E. (2004). Górskie krajobrazy Włoch w polskim piśmiennictwie geograficznym i podróżniczym XVIII wieku. In B. Rok, \& F. Wolański (Eds.), Staropolski ogląd świata (pp. 279-288). Wrocław: Wydawnictwo Uniwersytetu Wrocławskiego.

Kowalczyk, M.E. (2005). Obraz Włoch $w$ polskim piśmiennictwie geograficznym i podróżniczym osiemnastego wieku. Toruń: Wydawnictwo Adam Marszałek.

Kowalczyk, M.E. (2014). Polki w Wenecji w drugiej połowie XVIII wieku. Zapiski z podróży Teofili z Radziwiłłów Morawskiej i Katarzyny z Sosnowskich Platerowej. Italica Wratislaviensia, 5, 317-337. 
Kowalczyk, M.E. (2019). Zagraniczne podróże Polek w epoce oświecenia. Łomianki: Wydawnictwo LTW.

Łukaszewicz, J. (2021). La Padova di August Fryderyk Moszyński. Italica Wratislaviensia, 12 (1), 123-140.

Markiewicz, A. (2011). Podróże edukacyjne w czasach Jana III Sobieskiego. Peregrinationes Jablonovianae. Warszawa: Wydawnictwo DiG.

Mączak, A. (2001). Peregrynacje, wojaże, turystyka. Warszawa: Książka i Wiedza.

Ordzowiały-Grzegorczyk, K. (2011). Tomasz Kajetan Węgierski jako świadek narodzin amerykańskiej państwowości. Perspektywy Kultur, 5 (2), 71-94.

Puchowski, K. (2007). Jezuickie kolegia szlacheckie Rzeczypospolitej Obojga Narodów. Studium z dziejów edukacji elit. Gdańsk: Wydawnictwo Uniwersytetu Gdańskiego.

Sajkowski, A. (1973). Włoskie przygody Polaków. Warszawa: PIW.

Siemieński, L. (1850). Podróże i pamiętniki T.K. Węgierskiego. Biblioteka Warszawska, 4, 474-495.

Stasiewicz, P. (2012). Poezja Tomasza Kajetana Wegierskiego. Białystok: Wydawnictwo Uniwersytetu w Białymstoku.

Tenenti, A. (1985). Venezia eil Veneto nelle pagine dei viaggiatori stranieri (1650-1790). Vicenza: Neri Pozza Editore.

Wdowik, A. (2014). Polacy w Spa na trasie podróży w drugiej połowie XVIII wieku. In A. Roćko (Ed.), Polski Grand Tour w XVIII i początkach XIX wieku (pp. 217-228). Warszawa: Muzeum Pałacu Króla Jana III w Wilanowie.

Węgierski, T.K. (2002). Wiersze wybrane (edited by K. Bocian). Kraków: Universitas.

Węgierski, T.K. (2007). Organy. Poema heroikomiczne (edited by A. Norkowska). Warszawa: Instytut Badań Literackich.

Woźniakowski, J. (1995). Góry niewzruszone. O różnych wyobrażeniach przyrody $w$ dziejach nowożytnej kultury europejskiej. Kraków: Społeczny Instytut Wydawniczy Znak.

Wrześniak, M. (2010). Roma Santa, Fiorenza Bella. Dzieła sztuki w pamiętnikach polskich podróżników do Włoch w XVI i XVII stuleciu. Warszawa: Wydawnictwo Uniwersytetu Kardynała Stefana Wyszyńskiego.

Wrześniak, M. (2013). Florencja-muzeum. Miasto i jego sztuka w oczach polskich podróżników. Kraków: Universitas. 


\title{
Ziontek, A. (2016). Ślady poetyckiego dialogu? Dwa wiersze - Tomasza Kaje- tana Węgierskiego i Michała Kazimierza Ogińskiego. In A. Borkowski, \& A. Czyż (Eds.), Prywatne i publiczne $w$ tekstach kultury: studia (pp. 123-149). Siedlce: Pracownia Wydawnicza Wydziału Humani- stycznego Uniwersytetu Przyrodniczo-Humanistycznego.
}

\begin{abstract}
Riassunto: Nella ricca produzione in prosa di Tomasz Kajetan Węgierski (1755-1787), poeta e satirico di talento, occupa un posto particolare il diario redatto in francese in forma epistolare durante il suo viaggio in Italia nel 1779. La sua destinataria era una certa Julia, amica parigina e amante del poeta. Le lettere italiane di Węgierski si sono conservate nelle collezioni della Sezione Manoscritti della Biblioteca Jagellonica di Cracovia, purtroppo come copia incompleta. Disponiamo di sette lettere intere, scritte nel novembre e nel dicembre del 1779 da Verona, Vicenza, Padova e Venezia, nonché della parte iniziale di un'ottava, anch'essa datata a Venezia. Inoltre, ci sono pervenuti i commenti di Węgierski ad alcune lettere mancanti, di cui quattro riguardano la città sulla laguna e uno Ferrara. Si è conservata anche una parte di una lettera scritta da Roma e dedicata alle catacombe. Dal suo contenuto veniamo a sapere che il poeta intendeva tornare nella città di San Marco per assistere alla cerimonia dello Sposalizio di Venezia con il mare, che cadeva in concomitanza con la festa dell'Ascensione. Scopo del presente articolo è presentare le ragioni che indussero il poeta nel 1779 a lasciare la Confederazione polacco-lituana e a recarsi in Italia, nonché svolgere un'analisi delle sue lettere, scritte durante il soggiorno nel Veneto. Vale la pena di sottolineare che questa regione costituiva una semplice tappa, per quanto significativa, lungo l'itinerario che conduceva Węgierski nella capitale dello Stato Pontificio. La Venezia Euganea affascinava il polacco, lo sorpendeva e scioccava. Nelle sue note di viaggio, piene di aneddoti e facezie, troviamo ampie descrizioni della storia e della cultura di Verona, Vicenza, Padova e Venezia, estesi ritratti dei suoi abitanti e delle loro usanze, nonché le più svariate informazioni sulla vita quotidiana, raccolte lungo la via. La narrazione di Węgierski, pur differendo per diversi aspetti dalle descrizioni di viaggio dei suoi predecessori, è piuttosto tipica dell'epoca in cui viveva.
\end{abstract}

Parole chiave: Tomasz Kajetan Węgierski, Veneto, Illuminismo, viaggi, lettere, vita quotidiana

\section{Traduzione dal polacco di Maurizio Mazzini}

